\title{
Acute subdural hematoma in the elderly: outcome analysis in a retrospective multicentric series of 213 patients
}

\author{
Gianluca Trevisi, MD, ${ }^{1}$ Carmelo Lucio Sturiale, MD, PhD, ${ }^{2}$ Alba Scerrati, MD, 3,4 \\ Oriela Rustemi, MD, ${ }^{5}$ Luca Ricciardi, MD, ${ }^{6}$ Fabio Raneri, MD, ${ }^{5}$ Alberto Tomatis, MD, ${ }^{1}$ \\ Amedeo Piazza, MD, ${ }^{6}$ Anna Maria Auricchio, MD, ${ }^{2}$ Vito Stifano, MD, ${ }^{2}$ Carmine Romano, MD, ${ }^{3}$ \\ Pasquale De Bonis, MD, PhD, ${ }^{3,4}$ and Annunziato Mangiola, MD ${ }^{1,7}$
}

\begin{abstract}
${ }^{1}$ Neurosurgical Unit, Ospedale Santo Spirito, Pescara; ${ }^{2}$ Department of Neurosurgery, Fondazione Policlinico Universitario A. Gemelli IRCSS, Rome; ${ }^{3}$ Department of Neurosurgery, S. Anna University Hospital, Ferrara; ${ }^{4}$ Department of Morphology, Surgery and Experimental Medicine, University of Ferrara, Ferrara; ${ }^{5}$ OC Neurochirurgia 1, Azienda ULSS 8 Berica Ospedale San Bortolo, Vicenza; ${ }^{6} U O C$ di Neurochirurgia, Azienda Ospedaliera Sant'Andrea, Dipartimento NESMOS, Sapienza-Roma; and 'Department of Neurosciences, Imaging and Clinical Sciences, "G. D’Annunzio" University, Chieti, Italy
\end{abstract}

OBJECTIVE The objective of this study was to analyze the risk factors associated with the outcome of acute subdural hematoma (ASDH) in elderly patients treated either surgically or nonsurgically.

METHODS The authors performed a retrospective multicentric analysis of clinical and radiological data on patients aged $\geq 70$ years who had been consecutively admitted to the neurosurgical department of 5 Italian hospitals for the management of posttraumatic ASDH in a 3-year period. Outcome was measured according to the Glasgow Outcome Scale (GOS) at discharge and at 6 months' follow-up. A GOS score of 1-3 was defined as a poor outcome and a GOS score of 4-5 as a good outcome. Univariate and multivariate statistics were used to determine outcome predictors in the entire study population and in the surgical group.

RESULTS Overall, 213 patients were admitted during the 3-year study period. Outcome was poor in 135 (63\%) patients, as $65(31 \%)$ died during their admission, $33(15 \%)$ were in a vegetative state, and $37(17 \%)$ had severe disability at discharge. Surgical patients had worse clinical and radiological findings on arrival or during their admission than the patients undergoing conservative treatment. Surgery was performed in $147(69 \%)$ patients, and 114 (78\%) of them had a poor outcome. In stratifying patients by their Glasgow Coma Scale (GCS) score, the authors found that surgery reduced mortality but not the frequency of a poor outcome in the patients with a moderate to severe GCS score. The GCS score and midline shift were the most significant predictors of outcome. Antiplatelet drugs were associated with better outcomes; however, patients taking such medications had a better GCS score and better radiological findings, which could have influenced the former finding. Patients with fixed pupils never had a good outcome. Age and Charlson Comorbidity Index were not associated with outcome.

CONCLUSIONS Traumatic ASDH in the elderly is a severe condition, with the GCS score and midline shift the stronger outcome predictors, while age per se and comorbidities were not associated with outcome. Antithrombotic drugs do not seem to negatively influence pretreatment status or posttreatment outcome. Surgery was performed in patients with a worse clinical and radiological status, reducing the rate of death but not the frequency of a poor outcome.

https://thejns.org/doi/abs/10.3171/2020.7.FOCUS20437

KEYWORDS acute subdural hematoma; antithrombotic drugs; comorbidity; elderly; surgery; traumatic brain injury

$\mathrm{G}$ IVEN the aging population, especially in some countries, the incidence of traumatic acute subdural hematoma (ASDH) in the elderly is an increasing clinical scenario, ${ }^{1}$ often presenting a dramatic picture with poor chances of recovery regardless of the treatment. ${ }^{2}$ Neither clear-cut evidence nor guidelines are currently available to help neurosurgeons detect those elderly patients who can benefit from prompt surgical treatment. ${ }^{3}$ In general, ASDH surgery is indicated in cases of impaired consciousness, neurological symptoms, hematoma thickness $>1 \mathrm{~cm}$, or midline shift $>5 \mathrm{~mm}$. However, age has been recognized as a major prognostic factor in brain trau-

ABBREVIATIONS ASDH = acute subdural hematoma; BMT = best medical treatment; $\mathrm{CCI}=$ Charlson Comorbidity Index; $\mathrm{CSDH}=$ chronic subdural hematoma; $\mathrm{DC}=$ decompressive craniectomy; GCS = Glasgow Coma Scale; GOS = Glasgow Outcome Scale.

SUBMITTED May 27, 2020. ACCEPTED July 17, 2020.

INCLUDE WHEN CITING DOI: 10.3171/2020.7.FOCUS20437. 
ma, ${ }^{4-8}$ and a mortality rate up to $80 \%$ has been reported in elderly patients who have undergone surgical evacuation of an ASDH. ${ }^{8}$ Besides death, a vegetative state or severe residual disability has often been reported in these patients, all regarded as poor outcomes., ${ }^{2,9,10}$

Even with an awareness of these adverse events, some factors such as family pressure, medico-legal aspects, and intradepartmental reputation could influence whether an aggressive surgical approach is followed despite the expectation of a poor outcome. ${ }^{11}$ Among the outcome predictors, level of consciousness according to the Glasgow Coma Scale (GCS) score, ASDH thickness, and amount of midline shift have been recognized as the most important, , ,6,7,12-14 while the role of antithrombotic therapy ${ }^{15,16}$ and the presence of comorbidities ${ }^{17,18}$ remain controversial.

In this multicentric retrospective study, we assessed the clinical and radiological data of elderly patients (age $\geq 70$ years) who had been consecutively admitted for an ASDH at 5 Italian tertiary care referral neurosurgical centers in a 3-year period. The aim of this study was to analyze the variables associated with outcomes, defined as good or poor according to the Glasgow Outcome Scale (GOS), in an entire ASDH population and in a surgically treated subgroup, both at discharge and at 6 months' follow-up.

\section{Methods}

\section{Population and Treatment}

We included all patients aged $\geq 70$ years who had been consecutively admitted to the neurosurgical department or ICU of 5 Italian hospitals for the management of posttraumatic ASDH between January 1, 2016, and December 31, 2019. We retrospectively reviewed all clinical records and radiological data archived with the PACS. All the neurosurgical units participating in the study have a $24 / 7$ service and are the reference unit of territories with an average population above 500,000 persons. ASDH was defined as a hyperdense subdural collection on CT. Patients with clear radiological evidence of an ASDH collection within a preexisting chronic subdural hematoma (CSDH) or with another associated intracranial lesion overwhelming the clinical significance of the ASDH collection were excluded.

No guidelines on the treatment of ASDH in elderly patients are currently available. As a rule, indications for surgery were based on one or more of the following: impaired consciousness, neurological symptoms, ASDH thickness > $1 \mathrm{~cm}$, and midline shift $>5 \mathrm{~mm}$. However, some patients presenting with one or more of these factors may have been treated conservatively if surgery was deemed not beneficial after taking into account the patient's clinical and radiological status, comorbidities, use of medications, age, and family consultation. Medical treatment included one or more of the following according to the patient's needs: hydration, steroids, antiepileptic drugs, head elevation, sedation, osmotic diuretic agents, hyperosmolar solutions, and ventilatory and cardiovascular support. These were also used in the postoperative period for surgical patients.

\section{Data Collection}

We attempted to collect the following information on all patients: age and sex; Charlson Comorbidity Index
(CCI); history of arterial hypertension and use of antithrombotic drugs; mechanism of injury; neurological status on arrival and during admission including the GCS score, pupillary size and reactivity to light, neurological deficits, and seizures; head CT parameters such as ASDH side and thickness, midline shift and presence of other posttraumatic lesions on the first $\mathrm{CT}$ and on subsequent (control) CT; and medical management such as urgent coagulopathy correction, best medical treatment (BMT), or surgical treatment of the ASDH.

In surgical cases, we also collected information about surgical timing with respect to a patient's hospital arrival $(<6,12-24,24-48,48-72,>72$ hours after admission to the Emergency Department, treatment at hematoma chronicization); size of the craniotomy in square centimeters; whether a primary (during a first surgery) or a secondary (at a redo surgery, with or without additional evacuation of the hematoma) decompressive craniectomy (DC) was performed; amount of hematoma evacuation measured at the early (within 48 hours after surgery) postoperative CT scanning $(100 \%, \geq 50 \%,<50 \%$, unchanged/increased hematoma); and clinical and radiological postoperative complications.

For all patients, we also collected data on length of the hospital stay in days, site of discharge (home, medical ward, rehabilitation center, elderly care center, peripheral hospital ICU), additional neurosurgical procedures related to the ASDH after initial discharge, clinical and radiological status at the last follow-up, and nonneurosurgical causes of death after discharge.

\section{Outcome Measures}

Outcome was measured according to the GOS: death, vegetative status, severe disability, moderate disability, or good recovery. GOS scores of 1-3 (death, vegetative state, severe disability) were considered as a poor outcome and scores of 4-5 (moderate disability, good recovery) as a good outcome. Outcome was measured at two time points: at hospital discharge and at 6 months' follow-up.

\section{Statistical Analysis}

An independent-samples Student t-test (Welch test in case of violated equal variance assumption) for quantitative variables and a chi-square test for qualitative variables were used to assess the differences between patients with different outcomes (good vs poor) in the entire population and between patients who had undergone surgery or BMT. Significant variables on univariate analysis were included as independent variables in a multivariate binomial logistic regression to analyze their association with the dependent variable outcome (good vs poor) both in the overall study population and in a subgroup analysis of the surgical group. If patients experienced clinical or radiological deterioration, a worse GCS level, worse ASDH thickness, and midline shift were considered if not otherwise indicated.

A logistic regression was also conducted to detect the association between clinical and radiological worsening and the independent variables age, admission GCS, CCI, ASDH thickness, midline shift, and use of antithrombotic drugs. 
The significance level was set at 0.05 . Statistical analysis was performed using JASP version 0.11.1.

\section{Results \\ Patient Demographics and Characteristics}

During the considered 3-year period, 213 elderly patients (mean age $80 \pm 7$ years, 112 males) were admitted for the management of ASDH at the participating centers. The majority (49\%) had a mild GCS score on admission. An early (within 72 hours) GCS score decrease related to the ASDH was documented in 37 (17\%) patients, while radiological worsening of either ASDH thickness or midline shift was seen in $43(20 \%)$ on a CT scan obtained after the initial CT scan.

The mechanism of injury was a ground-level fall in 111 (52\%) patients, a fall from a height $>2 \mathrm{~m}$ in 15 (7\%), and a motor vehicle accident in $10(5 \%)$; other mechanisms of injury or a clear trauma with no further details occurred in the remaining 77 (36\%) patients.

The majority of patients (69\%) were on antithrombotic drugs or had a coagulopathy on admission: $68(32 \%)$ patients were on a single antiplatelet drug, $65(31 \%)$ on anticoagulants, 7 (3\%) on dual antiplatelet therapy, $6(3 \%)$ on combined anticoagulant and antiplatelet therapy, and 1 $(0.5 \%)$ had a low platelet count. The most common antiplatelet agent was acetylsalicylic acid, taken by 65 patients (80\% of those on antiplatelets). Vitamin K antagonists were the most common anticoagulants (46 patients, $65 \%$ of those on anticoagulants).

The most common comorbidities were coronary artery disease (42\% of patients), arterial hypertension (35\%), diabetes (31\%), chronic atrial fibrillation (21\%), and dementia (20\%). The mean CCI was 5.3 \pm 1.7 . Descriptive results are summarized in Tables 1-3.

\section{Treatment}

BMT was offered to 66 patients (31\%). Most patients in the BMT group had a GCS score $\geq 13$ on arrival (77\%) and maintained their good neurological status during admission, as a GCS score decrease was recorded in only 3 $(5 \%)$ of them. Patients in the BMT group were significantly older and more frequently took antithrombotic drugs than the surgical patients, whereas urgent coagulopathy correction was significantly more frequent in patients undergoing surgical treatment.

Moreover, the 147 patients undergoing surgery were in worse clinical and radiological condition than the patients treated nonsurgically. The majority of them were in the moderate $(24 \%)$ or severe $(40 \%)$ GCS group before surgery, and pupillary abnormalities were documented in 48 of the surgical patients (33\%), with pupils fixed and dilated in 11 of them. A GCS score decrease after arrival was significantly more frequent in the surgical group than in the BMT group. Hematoma thickness and midline shift were also significantly greater in the surgical group, both on arrival and on early pretreatment CT scans. These significant radiological differences between treatment groups were particularly evident among the patients with a mild GCS level (Table 3).

No significant difference between the two treatment groups was seen regarding the side of the hematoma, mean CCI, frequency of other associated intracranial lesions, or neurological deficits or seizures on admission.

Standalone ICP monitoring was used in only one case in the BMT group, whereas postoperative ICP was regularly monitored in surgical cases with decreased consciousness. Delayed surgery for a CSDH was performed in $14(21 \%)$ patients in the BMT group. Table 1 summarizes demographic differences in the two treatment groups.

\section{Surgical Group}

Primary DC was performed in 14 of the 147 surgical patients (10\%). An early postoperative CT scan was available in 140 patients, as 7 had unstable conditions rapidly evolving toward death postoperatively. A complete hematoma evacuation was seen on 78 scans (56\% of patients with available scans), an evacuation of more than half of the hematoma volume on 49 (35\%), an evacuation of less than half on $10(7 \%)$, and an unchanged or increased hematoma on $3(2 \%)$. Patients with complete hematoma evacuation had a statistically significantly thinner ASDH than the patients with partial (> 50\%) evacuation: 15.6 vs $18.9 \mathrm{~mm}(\mathrm{p}=0.03)$. There was no statistically significant relationship between extent of evacuation and preoperative midline shift or GCS level.

Postoperative complications were recorded in 72 surgical patients (49\%), most commonly rebleeding in the subdural and/or intracerebral space on the delayed postoperative CT scan (19\% of complications), seizures (15\%), cardiopulmonary failure (13\%), infection (10\%), and stroke $(8 \%)$. A combination of these was seen in the great majority of the remaining cases of postoperative complications. Patients with postoperative complications had statistically significantly greater mean preoperative midline shift than the patients with no postoperative complications (11.9 vs $9.3 \mathrm{~mm}, \mathrm{p}=0.02$ ). Redo surgery for ASDH evacuation was performed in 6 patients and was associated with DC in 3 of the 6 cases. No standalone secondary DCs were performed.

\section{Outcome}

\section{Entire Study Population}

Overall, outcome was poor in $135(63 \%)$ patients, and $65(31 \%)$ patients died during their hospital stay. At discharge, $48(23 \%)$ patients had a good recovery, $30(14 \%)$ a moderate disability, $37(17 \%)$ a severe disability, and 33 $(15 \%)$ were in a vegetative state. Forty-six $(22 \%)$ patients were discharged to home, $42(20 \%)$ to a rehabilitation center, $25(12 \%)$ to a peripheral hospital ICU, $22(10 \%)$ to eldercare, 5 (2\%) to a medical ward, and 8 (4\%) had missing data or other discharge locations. At 6 months' follow-up, $71(48 \%)$ of the 148 patients who had been alive at discharge maintained a good outcome, representing $33 \%$ of the total series sample.

\section{BMT Group and Surgery Group}

The two treatment groups were not directly comparable, as BMT was offered almost exclusively to patients with a better clinical and radiological status. Indeed, a statistically significantly higher rate of patients with a good outcome 
TABLE 1. Descriptive results for 213 elderly patients treated for ASDH

\begin{tabular}{|c|c|c|c|c|}
\hline Variable & BMT & ASDH Surgery & Total & $\mathrm{p}$ Value \\
\hline No. of patients & $66(31)$ & $147(69)$ & 213 & \\
\hline Mean age in yrs (min-max) & $83 \pm 7(71-99)$ & $78 \pm 6(70-92)$ & $80 \pm 7(70-99)$ & $<0.001^{*}$ \\
\hline Male sex & $27(41)$ & $85(58)$ & $112(53)$ & $0.02^{*}$ \\
\hline Lt-sided hematoma & $40(61)$ & $71(48)$ & $111(52)$ & 0.2 \\
\hline Mean $\mathrm{CCl}$ & $5.4 \pm 1.6$ & $5.2 \pm 1.7$ & $5.3 \pm 1.7$ & 0.5 \\
\hline Antithrombotic drugs & $55(83)$ & $92(63)$ & $147(69)$ & $0.002^{*}$ \\
\hline Urgent coagulopathy correction & $2(3)$ & $30(20)$ & $32(15)$ & $<0.001^{*}$ \\
\hline \multicolumn{5}{|l|}{ GCS score at arrival } \\
\hline Mean & $13 \pm 3$ & $10 \pm 3$ & $11 \pm 4$ & $<0.001^{*}$ \\
\hline Mild, 13-15 & $51(77)$ & $53(36)$ & $104(49)$ & \multirow{3}{*}{$<0.001^{*}$} \\
\hline Moderate, 9-12 & $8(12)$ & $35(24)$ & $43(20)$ & \\
\hline Severe, 3-8 & $7(11)$ & $59(40)$ & $66(31)$ & \\
\hline Neurological deficits at admission & $21(32)$ & $51(35)$ & $72(34)$ & 0.7 \\
\hline Seizures at admission & $6(9)$ & $7(5)$ & $13(6)$ & 0.2 \\
\hline \multicolumn{5}{|l|}{ Pupillary reaction to light } \\
\hline Normal & $57(86)$ & $84(57)$ & $142(67)$ & \multirow{4}{*}{$0.004^{*}$} \\
\hline Anisocoric & $7(11)$ & $37(25)$ & $44(21)$ & \\
\hline Fixed & $1(2)$ & $11(7)$ & $12(6)$ & \\
\hline Missing data & & $15(10)$ & & \\
\hline \multicolumn{5}{|l|}{ Initial CT scan } \\
\hline Mean ASDH thickness in mm (min-max) & $13 \pm 7(1-33)$ & $16 \pm 7(1-38)$ & $14 \pm 7(1-38)$ & $<0.008^{*}$ \\
\hline Mean MLS in mm (min-max) & $5 \pm 6(0-29)$ & $9 \pm 6(0-30)$ & $8 \pm 6(0-38)$ & $<0.001^{*}$ \\
\hline Other posttraumatic intracranial lesions & $24(36)$ & $62(42)$ & $86(40)$ & 0.4 \\
\hline GCS decrease w/in 72 hrs from admission $†$ & $3(5)$ & $34(23)$ & $37(17)$ & $<0.001^{*}$ \\
\hline \multicolumn{5}{|l|}{ GCS score during admission† } \\
\hline Mean score & $13 \pm 3$ & $8 \pm 4$ & $10 \pm 5$ & $<0.001^{*}$ \\
\hline Mild, 13-15 & $49(74)$ & $33(22)$ & $82(38)$ & \multirow{3}{*}{$<0.001^{*}$} \\
\hline Moderate, 9-12 & $9(14)$ & $36(24)$ & $45(21)$ & \\
\hline Severe, 3-8 & $8(12)$ & $78(53)$ & $86(40)$ & \\
\hline New-onset/worsening deficits or seizures & $11(17)$ & $29(20)$ & $40(19)$ & 0.6 \\
\hline \multicolumn{5}{|l|}{ Changes on early pretreatment CT scans† } \\
\hline Radiological worsening & $15(23)$ & $28(19)$ & $43(20)$ & 0.5 \\
\hline Worsening of ASDH thickness & $14(21)$ & $26(18)$ & $40(19)$ & 0.5 \\
\hline Worsening of MLS & $11(17)$ & $28(19)$ & $39(18)$ & 0.7 \\
\hline Mean ASDH thickness in mm (min-max) & $14 \pm 8(1-33)$ & $17 \pm 6(3-38)$ & $16 \pm 7(1-38)$ & $<0.001^{*}$ \\
\hline Mean MLS in mm (min-max) & $6 \pm 6(0-29)$ & $11 \pm 6(0-30)$ & $9 \pm 6(0-30)$ & $<0.001^{*}$ \\
\hline Mean hospital LOS in days (min-max) & $13 \pm 12(0-64)$ & $18 \pm 21(0-95)$ & $17 \pm 19(0-95)$ & $0.04^{*}$ \\
\hline Death during admission & $14(21)$ & $51(35)$ & $65(31)$ & $0.048^{*}$ \\
\hline Good outcome at discharge $\ddagger$ & $45(68)$ & $33(22)$ & $78(37)$ & $<0.001^{*}$ \\
\hline Additional deaths w/in 6 mos of ASDH & $7(11)$ & $13(9)$ & $20(9)$ & 0.4 \\
\hline Good outcome at 6 mos $\ddagger$ & $39(59)$ & $32(22)$ & $71(33)$ & $0.003^{*}$ \\
\hline Surgery for CSDH & $14(21)$ & 0 & $14(7)$ & $<0.001^{*}$ \\
\hline
\end{tabular}

FU = follow-up; LOS = length of stay; MLS = midline shift.

Values are expressed as the mean \pm standard deviation or as number of patients $(\%)$, unless indicated otherwise.

* Statistically significant.

$\dagger$ For the surgery group, this refers to changes that occurred before surgical treatment in cases in which surgery was not performed soon after hospital arrival and the initial CT scan.

‡ Grouped according to GOS score: $1-3$, poor outcome; $4-5$, good outcome. 
TABLE 2. Analytic description of results according to the GOS at discharge

\begin{tabular}{|c|c|c|c|c|c|c|c|}
\hline Variable & $\begin{array}{c}\text { Good } \\
\text { Recovery }\end{array}$ & $\begin{array}{l}\text { Moderate } \\
\text { Disability }\end{array}$ & $\begin{array}{c}\text { Severe } \\
\text { Disability }\end{array}$ & $\begin{array}{l}\text { Vegetative } \\
\text { State }\end{array}$ & Death & Total & $p$ Value \\
\hline No. of patients & 48 & 30 & 37 & 33 & 65 & 213 & \\
\hline GCS level at admission & & & & & & & $<0.001^{*}$ \\
\hline Mild & 43 & 24 & 11 & 8 & 18 & 104 & \\
\hline Moderate & 5 & 4 & 15 & 7 & 12 & 43 & \\
\hline Severe & 0 & 2 & 11 & 18 & 35 & 66 & \\
\hline GCS level during admission & & & & & & & $<0.001^{*}$ \\
\hline Mild & 39 & 18 & 8 & 6 & 11 & 82 & \\
\hline Moderate & 8 & 5 & 13 & 6 & 13 & 45 & \\
\hline Severe & 1 & 7 & 16 & 21 & 41 & 86 & \\
\hline Pupillary reaction to light & & & & & & & $<0.001^{*}$ \\
\hline Normal & 47 & 26 & 29 & 14 & 26 & 142 & \\
\hline Anisocoric & 1 & 4 & 8 & 10 & 21 & 44 & \\
\hline Fixed & 0 & 0 & 0 & 5 & 7 & 12 & \\
\hline Timing of surgery after admission in hrs & & & & & & & $<0.001^{*}$ \\
\hline$<6$ & 3 & 2 & 8 & 5 & 18 & 36 & \\
\hline $6-12$ & 3 & 5 & 8 & 2 & 9 & 27 & \\
\hline $12-24$ & 4 & 6 & 9 & 21 & 14 & 54 & \\
\hline $24-48$ & 0 & 2 & 1 & 0 & 3 & 6 & \\
\hline $48-72$ & 1 & 2 & 2 & 1 & 2 & 8 & \\
\hline$>72$ & 3 & 2 & 3 & 3 & 5 & 16 & \\
\hline Surgery at chronicization & 8 & 2 & 2 & 1 & 1 & 14 & \\
\hline No surgery & 26 & 9 & 4 & 0 & 13 & 52 & \\
\hline Neurological deficits/seizures & & & & & & & $<0.001^{*}$ \\
\hline No & 32 & 11 & 11 & 23 & 35 & 112 & \\
\hline Yes & 16 & 19 & 26 & 10 & 30 & 101 & \\
\hline Antithrombotic drugs & & & & & & & $0.008^{*}$ \\
\hline No & 7 & 6 & 13 & 16 & 24 & 66 & \\
\hline Yes & 41 & 24 & 24 & 17 & 41 & 147 & \\
\hline Mean ASDH thickness in $\mathrm{mm}$ & $12.3 \pm 6.4$ & $14.5 \pm 5.9$ & $17.5 \pm 6.2$ & $15.6 \pm 5.9$ & $19.2 \pm 7.5$ & $16 \pm 7.1$ & $<0.001^{*}$ \\
\hline Mean MLS in mm & $4.7 \pm 3.8$ & $6.8 \pm 4.2$ & $9.8 \pm 6.1$ & $10.8 \pm 6.2$ & $12.8 \pm 6.4$ & $9.2 \pm 6.3$ & $<0.001^{*}$ \\
\hline
\end{tabular}

TABLE 3. Outcome according to GCS level during admission and treatment

\begin{tabular}{|c|c|c|c|c|c|c|c|c|c|}
\hline \multirow[b]{2}{*}{ GCS Level } & \multirow[b]{2}{*}{$\begin{array}{c}\text { Mean } \\
\text { Age (yrs) }\end{array}$} & \multirow[b]{2}{*}{$\begin{array}{c}\text { Mean ASDH } \\
\text { Thickness (mm) }\end{array}$} & \multirow[b]{2}{*}{$\begin{array}{c}\text { Mean } \\
\text { MLS (mm) }\end{array}$} & \multicolumn{2}{|c|}{ Survival } & \multirow[b]{2}{*}{$p$ Value } & \multicolumn{2}{|c|}{ Outcome } & \multirow[b]{2}{*}{$\mathrm{p}$ Value } \\
\hline & & & & $\begin{array}{c}\text { Alive } \\
(n=148[69 \%])\end{array}$ & $\begin{array}{c}\text { Death } \\
(n=65[31 \%])\end{array}$ & & $\begin{array}{c}\text { Good } \\
(n=78[37 \%])\end{array}$ & $\begin{array}{c}\text { Poor } \\
(n=135[63 \%])\end{array}$ & \\
\hline \multicolumn{10}{|l|}{ Mild } \\
\hline BMT $(n=49)$ & 82 & 11 & 4 & $46(94)$ & $3(6)$ & \multirow{2}{*}{$0.02^{*}$} & $43(88)$ & $6(12)$ & \multirow{2}{*}{$<0.001^{*}$} \\
\hline Surgery $(n=33)$ & 79 & 16 & 9 & $25(76)$ & $8(24)$ & & $14(42)$ & $19(58)$ & \\
\hline \multicolumn{10}{|l|}{ Moderate } \\
\hline BMT $(n=9)$ & 85 & 17 & 8 & $4(44)$ & $5(56)$ & \multirow{2}{*}{$0.04^{*}$} & $1(11)$ & $8(89)$ & \multirow{2}{*}{0.2} \\
\hline Surgery $(n=36)$ & 78 & 16 & 9 & $28(78)$ & $8(22)$ & & $12(33)$ & $24(67)$ & \\
\hline \multicolumn{10}{|l|}{ Severe } \\
\hline BMT $(n=8)$ & 90 & 26 & 17 & $2(25)$ & $6(75)$ & \multirow{2}{*}{0.1} & $1(13)$ & $7(88)$ & \multirow{2}{*}{0.7} \\
\hline Surgery $(n=78)$ & 78 & 19 & 14 & $43(55)$ & $35(45)$ & & $7(9)$ & $71(91)$ & \\
\hline
\end{tabular}

$\mathrm{n}=$ number of patients.

Values are expressed as the number of patients (\%), unless indicated otherwise. Percentages refer to the total number of patients in each row.

* Statistically significant. 
TABLE 4. Univariate analysis: variables related to outcome at discharge among the overall study population

\begin{tabular}{lccc}
\hline \multicolumn{1}{c}{ Variable } & $\begin{array}{c}\text { Good Outcome } \\
(\mathrm{n}=78)\end{array}$ & $\begin{array}{c}\text { Poor Outcome } \\
(\mathrm{n}=135)\end{array}$ & $\mathrm{p} \mathrm{Value}$ \\
\hline Mean age in yrs & $80.3 \pm 6.4$ & $79.6 \pm 6.5$ & 0.4 \\
\hline Mean CCI & $5.1 \pm 1.6$ & $5.5 \pm 1.8$ & 0.08 \\
\hline Mean GCS score & $12.8 \pm 3$ & $7.8 \pm 4.3$ & $<0.001^{*}$ \\
\hline $\begin{array}{l}\text { Mean ASDH thickness } \\
\text { in mm }\end{array}$ & $13.2 \pm 6.3$ & $18 \pm 6.9$ & $<0.001^{*}$ \\
\hline Mean MLS in mm & $5.5 \pm 4$ & $11.5 \pm 6.4$ & $<0.001^{*}$ \\
\hline Male sex & $35(44.9)$ & $77(57.0)$ & 0.09 \\
\hline Lt hemisphere hematoma & $45(57.7)$ & $66(48.9)$ & 0.4 \\
\hline Associated posttraumatic & $27(34.6)$ & $59(43.7)$ & 0.2 \\
lesions & & & \\
\hline Fixed pupils & $0(0)$ & $12(9.0)$ & $0.004^{*}$ \\
\hline Arterial hypertension $\dagger$ & $35(56.5)$ & $39(57.4)$ & 0.9 \\
\hline Antithrombotic drugs & $65(83.3)$ & $82(60.7)$ & $<0.001^{*}$ \\
\hline $\begin{array}{l}\text { Neurological } \\
\text { deficits/seizures }\end{array}$ & $35(44.9)$ & $66(48.9)$ & 0.6 \\
\hline GCS level $\leq 8$ & $8(10.3)$ & $78(57.8)$ & $<0.001^{*}$ \\
\hline ASDH surgery & $33(42.3)$ & $114(84.4)$ & $<0.001^{*}$ \\
\hline
\end{tabular}

$\mathrm{n}=$ number of patients.

Values are expressed as the mean \pm standard deviation or as the number of patients (\%), unless indicated otherwise.

${ }^{*}$ Statistically significant.

† Data available in 130 patients, 62 with a good outcome and 68 with a poor outcome.

(68\% vs $22 \%)$ and with a lower rate of death (21\% vs $35 \%)$ during hospital admission was seen in the BMT group.

On the other hand, the percentage of patients at the moderate and severe GCS levels who were alive at discharge was higher in the surgical group than in the BMT group, with the difference between the two treatment groups being significant for the moderate GCS level (Table 3). However, treatment groups were also heterogeneous in the moderate and severe GCS levels, whereas BMT was reserved for very elderly patients. Nonetheless, the two treatment groups showed no significant difference in terms of a good or poor outcome for patients with GCS score $<13$.

\section{Analysis of Outcome Predictors}

On univariate analysis, poor outcome predictors in the overall population were a lower GCS score, dilated fixed pupils, less use of antithrombotic drugs, increased ASDH thickness, and greater midline shift (Table 4).

Lower GCS score, greater midline shift, and use of antithrombotic drugs also showed a significant association with outcome in the subgroup analysis of patients who had undergone surgery (Table 5). In this group, outcome was not positively influenced by prompt surgical treatment, as only $15 \%$ of patients operated on within 6 hours from hospital arrival had a good outcome at discharge. However, this subset of patients had a statistically significantly lower mean GCS score on admission than the patients operated on after 6 hours $(p=0.004)$. Associated intracranial lesions, craniotomy size, and amount of hematoma evacua-
TABLE 5. Univariate analysis: outcome at discharge among the acute surgery group

\begin{tabular}{|c|c|c|c|}
\hline Variable & $\begin{array}{l}\text { Good Outcome } \\
\qquad(n=33)\end{array}$ & $\begin{array}{l}\text { Poor Outcome } \\
\quad(n=114)\end{array}$ & $p$ Value \\
\hline Mean age in yrs & $78.2 \pm 5.3$ & $78.2 \pm 5.6$ & 0.99 \\
\hline Mean $\mathrm{CCl}$ & $5.2 \pm 1.8$ & $5.2 \pm 1.7$ & 0.9 \\
\hline Mean GCS score & $10.7 \pm 3.6$ & $7.4 \pm 4.3$ & $<0.001^{\star}$ \\
\hline $\begin{array}{l}\text { Mean ASDH thickness } \\
\text { in } \mathrm{mm}\end{array}$ & $16.3 \pm 5$ & $17.7 \pm 6.9$ & 0.3 \\
\hline Mean MLS in mm & $7.5 \pm 3.9$ & $11.8 \pm 6.2$ & $<0.001^{*}$ \\
\hline $\begin{array}{l}\text { Mean size of craniotomy } \\
\text { in } \mathrm{cm}^{2}\end{array}$ & $37.7 \pm 27.6$ & $42.2 \pm 32.8$ & 0.5 \\
\hline Male sex & $18(55)$ & $67(59)$ & 0.7 \\
\hline Lt hemisphere & $18(55)$ & $53(46)$ & 0.6 \\
\hline Fixed pupils & 0 & $10(9)$ & 0.06 \\
\hline Arterial hypertension $\dagger$ & $15(75)$ & $27(57)$ & 0.2 \\
\hline Antithrombotic drugs & $27(82)$ & $65(57)$ & $0.01^{*}$ \\
\hline Coagulopathy correction & $6(18)$ & $18(16)$ & 0.7 \\
\hline $\begin{array}{l}\text { Neurological deficits/ } \\
\text { seizures }\end{array}$ & $20(61)$ & $51(45)$ & 0.1 \\
\hline GCS level $\leq 8$ & $7(21)$ & $71(62)$ & $<0.001^{*}$ \\
\hline $\begin{array}{l}\text { Surgery w/in } 6 \text { hrs of } \\
\text { admission }\end{array}$ & $5(15)$ & $31(27)$ & 0.2 \\
\hline Postop complications & $9(27)$ & $63(55)$ & $0.005^{\prime}$ \\
\hline \multicolumn{4}{|c|}{$\begin{array}{l}n=\text { number of patients. } \\
\text { Values are expressed as the mean } \pm \text { standard deviation or as the number of } \\
\text { patients }(\%) \text {, unless indicated otherwise. } \\
{ }^{*} \text { Statistically significant. } \\
\dagger \text { Data available in } 67 \text { patients, } 20 \text { with a good outcome and } 47 \text { with a poor } \\
\text { outcome. }\end{array}$} \\
\hline
\end{tabular}

tion were not associated with outcome. Postoperative complications significantly influenced outcomes $(p=0.005)$. However, a correlation between preoperative midline shift and postoperative complications was also documented.

Multivariate binomial logistic regression showed a significant association between poor prognosis and both a lower GCS score and greater midline shift in the overall population and in the surgical patients. Use of antithrombotic drugs showed an association with good outcome in the overall population but not in the surgical group (Table 6).

An analysis of the association between the category of antithrombotic drug, namely anticoagulant or antiplatelet, and outcome showed that patients using anticoagulants did not have significantly different outcomes (good/poor nor alive/dead) compared with those of patients who did not use antithrombotics. However, patients on antiplatelet drugs had a higher rate of good outcomes $(\mathrm{p}<0.001)$ than those not taking antiplatelet drugs. These data were also valid for patients on double antiplatelet therapy. Patients on antiplatelet therapy had a statistically significantly better GCS status ( $p$ 0.001) and less midline shift $(\mathrm{p}=0.04)$ than those not on antiplatelet therapy.

A decrease in the GCS score during admission was significantly associated with a lower age $(\mathrm{p}<0.001)$ and a higher GCS score at admission $(p=0.002)$. Radiological worsening had a significant association with less ASDH 
TABLE 6. Binomial logistic regression: outcomes at discharge according to GOS (good vs poor)

\begin{tabular}{lcccc}
\hline \multirow{2}{*}{ Dependent Variable } & Covariate & OR & $95 \% \mathrm{Cl}$ & $\mathrm{p}$ Value \\
\hline & GCS score & 0.824 & $0.735,0.924$ & $<0.001^{*}$ \\
\cline { 2 - 4 } & ASDH thickness & 1.037 & $0.961,1.119$ & 0.354 \\
\cline { 2 - 4 } $\begin{array}{l}\text { All patients: outcome at } \\
\text { discharge (good or poor) }\end{array}$ & MLS & 1.196 & $1.074,1.332$ & $0.001^{*}$ \\
\cline { 2 - 4 } & Fixed pupils & $3.6 \mathrm{e} 5$ & $0.000,>10 \mathrm{e} 10$ & 0.996 \\
\hline & Antithrombotic drugs & 0.322 & $0.120,0.864$ & $0.024^{*}$ \\
\hline \multirow{2}{*}{$\begin{array}{l}\text { ASDH surgery: outcome at } \\
\text { discharge (good or poor) }\end{array}$} & GCS score & 0.872 & $0.766,0.992$ & $0.037^{*}$ \\
\cline { 2 - 4 } & ASDH thickness & 1.004 & $0.913,1.103$ & 0.9 \\
\hline & MLS & 1.211 & $1.067,1.374$ & $0.003^{*}$ \\
\hline
\end{tabular}

Outcome level "poor" was coded as class 1. Covariates were included according to the univariate analysis results.

${ }^{*}$ Statistically significant.

thickness $(p=0.007)$ and less midline shift $(p=0.045)$. Antithrombotic drugs were not associated with clinical or radiological worsening.

\section{Discussion}

ASDH in elderly patients is often related to low-energy impacts from ground-level falls. ${ }^{2,12}$ Nonetheless, this series confirms a high rate of poor outcomes in patients aged $\geq$ 70 years who are admitted for an ASDH, as $31 \%$ died during their admission and another $32 \%$ were discharged in a vegetative state $(15 \%)$ or with a severe disability $(17 \%)$. Only $22 \%$ of patients could be discharged to home. No significant differences in neurological functional status were seen in surviving patients at 6 months after treatment.

The surgical management of elderly patients with ASDH is not standardized, as aged patients often have a comorbidity burden that limits surgical and anesthesiological management. Therefore, surgery is often precluded in patients who are believed to be in a "too good" or "too bad" clinical condition. For patients in better neurological conditions, a watchful waiting approach toward hematoma chronicization is sometimes pursued. ${ }^{19,20}$ In our series, this strategy could be put into effect in less than $7 \%$ of cases.

Although frailty has been recognized as a risk factor for a poor outcome in elderly patients with head trauma, ${ }^{5,17}$ age was not associated with outcome in our series. Neither was age identified as an independent prognostic factor in other studies., ${ }^{42}$ Similarly, we could not document differences in the mean CCI between the two outcome groups. The role of comorbidities in ASDH outcome in the elderly population is debated. ${ }^{2}$ A relatively high rate of good functional outcomes after craniotomy in elderly patients presenting with ASDH (41\% in GOS 4-5) has been reported after strict selection of patients with a Karnofsky Performance Status $>80$, independent daily living, and no diagnosis of dementia.? On the other hand, Won et al. did not document a significant increase in the odds of a poor outcome in patients with five or more comorbidities. ${ }^{1}$ Our results seem to confirm that in the setting of such an acute clinical and radiological scenario, the severity of intracranial abnormalities overcomes the burden of the systemic issues in affecting the survival and functional outcomes of elderly patients.
In line with several previous series, $, 2,21,22$ the GCS score and midline shift were factors significantly associated with the outcomes of our patients. Antithrombotic drugs were not associated with greater ASDH thickness and midline shift, nor with worsening clinical or radiological findings after initial assessment. This finding is in line with previous evidence in head trauma patients. ${ }^{23} \mathrm{~A}$ peculiar finding of our series was the "protective" role of antithrombotic drugs in the overall series population (OR $0.564, p=0.023$ ). While previous studies have reported an increased risk of a poor outcome in ASDH patients on anticoagulants, ${ }^{16,22}$ we did not find any difference in the distribution of outcomes in these patients and those not taking antithrombotic drugs. This may be attributable to an early pharmacological correction of the drug-related coagulopathy. Prompt international normalized ratio (INR) correction could have also reduced differences between warfarin and direct oral anticoagulants. Indeed, an increased frequency of good outcomes in patients on antiplatelets was found. However, since patients on antiplatelets had a better GCS score and less midline shift than the patients not on antiplatelets, the association between antiplatelet and outcome is likely strongly influenced by a better clinical and radiological starting point. However, we cannot find a specific reason for the better initial status of patients on antiplatelets, as it may occur by chance.

Another recognized sign associated with a poor outcome is pupil abnormalities, especially fixed dilated pupils. ${ }^{7,12}$ Our series showed that no patients with fixed pupils and only $5 / 44(11 \%)$ patients with anisocoric pupils had a good outcome. However, the small number of patients with fixed pupils probably limited the results of univariate and multivariate regression analyses in this regard.

\section{Role of Surgery}

Timing of surgery has often been regarded as an important variable associated with outcome in ASDH surgery. ${ }^{24-26}$ However, several studies have failed to demonstrate an association between the timing of surgery and outcome. ${ }^{27-29}$ Furthermore, no dedicated studies in the elderly population have been conducted. More importantly, differences in surgical timing may reflect different clinical severities. ${ }^{10,24}$ In our series, there was a range 
of timings for surgical treatment, going from less than 6 hours to 3 days after admission. This has been influenced by those patients who had a deterioration in their GCS score, the development or worsening of neurological deficits, or the worsening of radiological findings after initial assessment. However, early treatment did not influence outcome. This finding is probably related to a worse neurological and radiological picture on arrival, which influenced overall outcome.

The surgical group showed an overall worse outcome than the BMT group. However, patients undergoing surgery had a significant worse clinical and radiological status than the BMT patients. On the other hand, $35 \%$ of patients with a GCS score $<13$ who did not undergo surgery were alive at discharge compared to $62 \%$ in the surgical group (Table 3). Nonetheless, a good outcome (good recovery, moderate disability) was found in only 2 patients in the BMT group and in 19 in the surgical group, corresponding to $12 \%$ and $17 \%$ of patients at the severe/moderate GCS level in each treatment group, respectively. These data show that, especially at the moderate GCS level, while surgery is superior to BMT in avoiding death, the functional outcome of elderly patients with a GCS score $<13$ is poor despite surgical treatment.

The findings of the present and previous studies reinforce the notion that optimal treatment of an ASDH in an elderly patient remains controversial and can represent an ethical dilemma for the on-call neurosurgeon facing the decision of whether or not to operate. While in a less severe clinical/radiological scenario conservative treatment seems relatively safe, the odds of a poor outcome are high after surgical treatment in a patient with greater midline shift and a lower GCS score. Nonetheless, surgery seems the only reasonable attempt to avoid death in patients presenting with worse clinical/radiological findings. While an attitude of "we did all we could" may be attractive and believed to be safer from a medico-legal standpoint, ${ }^{11}$ the dismal outcomes reported here suggest paying careful attention to detailed information on expected outcomes in talking with family members and in trying to understand whether a patient clearly expressed his or her wishes previously.

\section{Study Limitations}

The main limitations of this study are its retrospective nature, which could have underestimated the number of elderly patients affected by ASDH, as patients in very good condition or dead before or soon after arrival could have been missed, as well as the heterogeneous distribution of patients among the two treatments (BMT or surgery). However, this distribution reflects the real-life scenario, as patients in worse clinical condition and with more severe radiological findings have more often undergone surgery than patients with a better clinical/radiological status. Moreover, the relatively low number of cases could limit the multivariate analysis results.

Finally, treatment indication and management of intracranial hypertension did not follow a common decision algorithm but was established by the local neurosurgical and ICU staff. However, no significant differences in outcomes among the 5 centers were seen, and our results are in line with the literature. This seems to emphasize that possible differences in raised ICP management have poor significance in terms of overall outcome, which is strongly influenced by the initial clinical/radiological status.

\section{Conclusions}

ASDH in patients aged $\geq 70$ years has a high rate of poor outcomes, as approximatively one-third of patients die during admission and another third is discharged in a vegetative state or with a severe disability. In this age group, however, age per se and CCI have no significant association with outcome. No significant differences in neurological functional status were seen in surviving patients 6 months after discharge. A poor GCS score and more pronounced midline shift have significant associations with a poor outcome. Patients with an abnormal pupillary response to light very rarely survive with a good outcome. The role of antithrombotic drugs is controversial, as they seem not to negatively influence hematoma volume and its tendency to grow, with antiplatelets even associated with better outcomes given a more favorable initial clinical/radiological condition. Surgery, generally reserved for patients in worse preoperative conditions, can significantly reduce the rate of death but not the frequency of patients with a poor outcome.

\section{References}

1. Won S-Y, Dubinski D, Brawanski N, et al. Significant increase in acute subdural hematoma in octo- and nonagenarians: surgical treatment, functional outcome, and predictors in this patient cohort. Neurosurg Focus. 2017;43(5):E10.

2. Evans LR, Jones J, Lee HQ, et al. Prognosis of acute subdural hematoma in the elderly: a systematic review. J Neurotrauma. 2019;36(4):517-522.

3. Bartek J Jr, Laugesen C, Mirza S, et al. Scandinavian Multicenter Acute Subdural Hematoma (SMASH) study: study protocol for a multinational population-based consecutive cohort. Neurosurgery. 2019;84(3):799-803.

4. Fountain DM, Kolias AG, Lecky FE, et al. Survival trends after surgery for acute subdural hematoma in adults over a 20-year period. Ann Surg. 2017;265(3):590-596.

5. Mushkudiani NA, Engel DC, Steyerberg EW, et al. Prognostic value of demographic characteristics in traumatic brain injury: results from the IMPACT study. J Neurotrauma. 2007;24(2):259-269.

6. Shimoda K, Maeda T, Tado M, et al. Outcome and surgical management for geriatric traumatic brain injury: analysis of 888 cases registered in the Japan Neurotrauma Data Bank. World Neurosurg. 2014;82(6):1300-1306.

7. Taussky P, Hidalgo ET, Landolt H, Fandino J. Age and salvageability: analysis of outcome of patients older than 65 years undergoing craniotomy for acute traumatic subdural hematoma. World Neurosurg. 2012;78(3-4):306-311.

8. Ushewokunze S, Nannapaneni R, Gregson BA, et al. Elderly patients with severe head injury in coma from the outset-has anything changed? Br J Neurosurg. 2004;18(6):604-607.

9. Petridis AK, Dörner L, Doukas A, et al. Acute subdural hematoma in the elderly; clinical and CT factors influencing the surgical treatment decision. Cent Eur Neurosurg. 2009;70(2): 73-78.

10. Sufaro Y, Avraham E, Alguyn F, et al. Unfavorable functional outcome is expected for elderly patients suffering from acute subdural hematoma even when presenting with preserved level of consciousness. J Clin Neurosci. 2019;67:167-171. 
11. Unterhofer C, Hartmann S, Freyschlag CF, et al. Severe head injury in very old patients: to treat or not to treat? Results of an online questionnaire for neurosurgeons. Neurosurg Rev. 2018;41(1):183-187.

12. Karibe H, Hayashi T, Hirano T, et al. Surgical management of traumatic acute subdural hematoma in adults: a review. Neurol Med Chir (Tokyo). 2014;54(11):887-894.

13. Herou E, Romner B, Tomasevic G. Acute traumatic brain injury: mortality in the elderly. World Neurosurg. 2015;83(6): 996-1001.

14. Whitehouse KJ, Jeyaretna DS, Enki DG, Whitfield PC. Head injury in the elderly: what are the outcomes of neurosurgical care? World Neurosurg. 2016;94:493-500.

15. Greuter L, Ullmann M, Mariani L, et al. Effect of preoperative antiplatelet or anticoagulation therapy on hemorrhagic complications in patients with traumatic brain injury undergoing craniotomy or craniectomy. Neurosurg Focus. 2019; 47(5):E3.

16. Won S-Y, Dubinski D, Bruder M, et al. Acute subdural hematoma in patients on oral anticoagulant therapy: management and outcome. Neurosurg Focus. 2017;43(5):E12.

17. Clegg A, Young J, Iliffe S, et al. Frailty in elderly people. Lancet. 2013;381(9868):752-762.

18. Lee HQ, Tan C, Tran V, et al. The utility of the modified Frailty Index in outcome prediction for elderly patients with acute traumatic subdural haematoma. J Neurotrauma. Published online July 8, 2020. doi:10.1089/neu.2019.6943

19. Gernsback JE, Kolcun JPG, Richardson AM, Jagid JR. Patientem fortuna adiuvat: the delayed treatment of surgical acute subdural hematomas-a case series. World Neurosurg. 2018; 120:e414-e420.

20. Kaestner S, van den Boom M, Deinsberger W. Frequency of and risk factors for chronification in traumatic acute subdural hematoma following conservative therapy. J Neurol Surg A Cent Eur Neurosurg. 2019;80(5):359-364.

21. Akbik OS, Starling RV, Gahramanov S, et al. Mortality and functional outcome in surgically evacuated acute subdural hematoma in elderly patients. World Neurosurg. 2019;126: e1235-e1241.

22. Raj R, Mikkonen ED, Kivisaari R, et al. Mortality in elderly patients operated for an acute subdural hematoma: a surgical case series. World Neurosurg. 2016;88:592-597.

23. Trevisi G, Scerrati A, Peppucci E, et al. What is the best timing of repeated CT scan in mild head trauma with an initially positive CT scan? World Neurosurg. 2018;118:e316-e322.

24. Bullock MR, Chesnut R, Ghajar J, et al. Surgical management of acute subdural hematomas. Neurosurgery. 2006; 58(3)(suppl):S16-S24, Si-Siv.

25. Seelig JM, Becker DP, Miller JD, et al. Traumatic acute subdural hematoma: major mortality reduction in comatose patients treated within four hours. N Engl J Med. 1981;304(25): $1511-1518$.
26. Wilberger JE Jr, Harris M, Diamond DL. Acute subdural hematoma: morbidity, mortality, and operative timing. J Neurosurg. 1991;74(2):212-218.

27. Kotwica Z, Brzeziński J. Acute subdural haematoma in adults: an analysis of outcome in comatose patients. Acta Neurochir (Wien). 1993;121(3-4):95-99.

28. Massaro F, Lanotte M, Faccani G, Triolo C. One hundred and twenty-seven cases of acute subdural haematoma operated on. Correlation between CT scan findings and outcome. Acta Neurochir (Wien). 1996;138(2):185-191.

29. Servadei F, Nasi MT, Giuliani G, et al. CT prognostic factors in acute subdural haematomas: the value of the 'worst' CT scan. Br J Neurosurg. 2000;14(2):110-116.

\section{Disclosures}

The authors report no conflict of interest concerning the materials or methods used in this study or the findings specified in this paper.

\section{Author Contributions}

Conception and design: Trevisi, Sturiale, Scerrati, Mangiola. Acquisition of data: Rustemi, Ricciardi, Raneri, Tomatis, Piazza, Auricchio, Stifano, Romano. Analysis and interpretation of data: Trevisi, Sturiale, Scerrati, Rustemi. Drafting the article: Trevisi. Critically revising the article: Trevisi, Sturiale, Ricciardi, Mangiola. Reviewed submitted version of manuscript: Trevisi, Sturiale, Scerrati, Rustemi, Ricciardi, Raneri, Tomatis, Piazza, Auricchio, Stifano, De Bonis, Mangiola. Approved the final version of the manuscript on behalf of all authors: Trevisi. Statistical analysis: Trevisi. Study supervision: De Bonis, Mangiola.

\section{Correspondence}

Gianluca Trevisi: Ospedale Santo Spirito, Pescara, Italy. trevisi. gianluca@gmail.com. 